\title{
On the Origin of the 21 Micron Feature in Post-AGB Stars
}

\author{
Sun Kwok \\ Department of Physics 83 Astronomy, University of Calgary, Calgary, \\ Canada
}

Kevin Volk

Department of Physics \& Astronomy, University of Calgary, Calgary, Canada

\author{
Bruce J. Hrivnak \\ Department of Physics $\mathcal{E}$ Astronomy, Valparaiso University, Valparaiso, \\ Indiana, USA
}

\begin{abstract}
The unidentified emission feature at $21 \mu \mathrm{m}$ is now observed in 12 sources, all being objects in transition between the asymptotic giant branch and planetary nebulae phases. The relations between the $21 \mu \mathrm{m}$ and other emission features, such as the PAH features and the broad $30 \mu \mathrm{m}$ feature, and the possible origins of the $21 \mu \mathrm{m}$ feature are discussed.
\end{abstract}

\section{Introduction}

Stars on the asymptotic giant branch (AGB) are classified as oxygen (M) or carbon (C) rich based on their photospheric abundances. Over 4000 and 700 stars have been found to show the silicate and the $\mathrm{SiC}$ features, respectively, in the IRAS Low Resolution Spectrometer (LRS) database and these stars are generally associated with $\mathrm{M}$ and $\mathrm{C}$ stars, respectively (Kwok, Volk \& Bidelman 1997). In addition to these features, strong emission features attributed to the PAH molecules are also found in planetary nebulae (PN). These molecules are believed to be synthesized in the AGB envelopes but are only excited by the UV radiation field during the $\mathrm{PN}$ stage. It therefore came as a surprise that proto-planetary nebulae (PPN), objects in transition between the AGB and PN phases, were found to show an entirely new, strong emission feature at $21 \mu \mathrm{m}$, a feature which is not seen in AGB stars or PN.

The unidentified emission feature at $21 \mu \mathrm{m}$ was first discovered in the IRAS LRS spectra of four carbon-rich post-AGB stars (Kwok, Volk \& Hrivnak 1989). Since the initial discovery, this feature has been detected by both airborne (Omont et al. 1995) and ground-based observations (Kwok, Hrivnak \& Geballe 1995; Justtanont et al. 1996).

To date, twelve $21 \mu \mathrm{m}$ sources have been identified. Their properties are summarized in Table 1. All these sources have relatively uniform properties. They are mostly carbon-rich $\mathrm{F}$ and $\mathrm{G}$ supergiants with strong infrared excesses. 


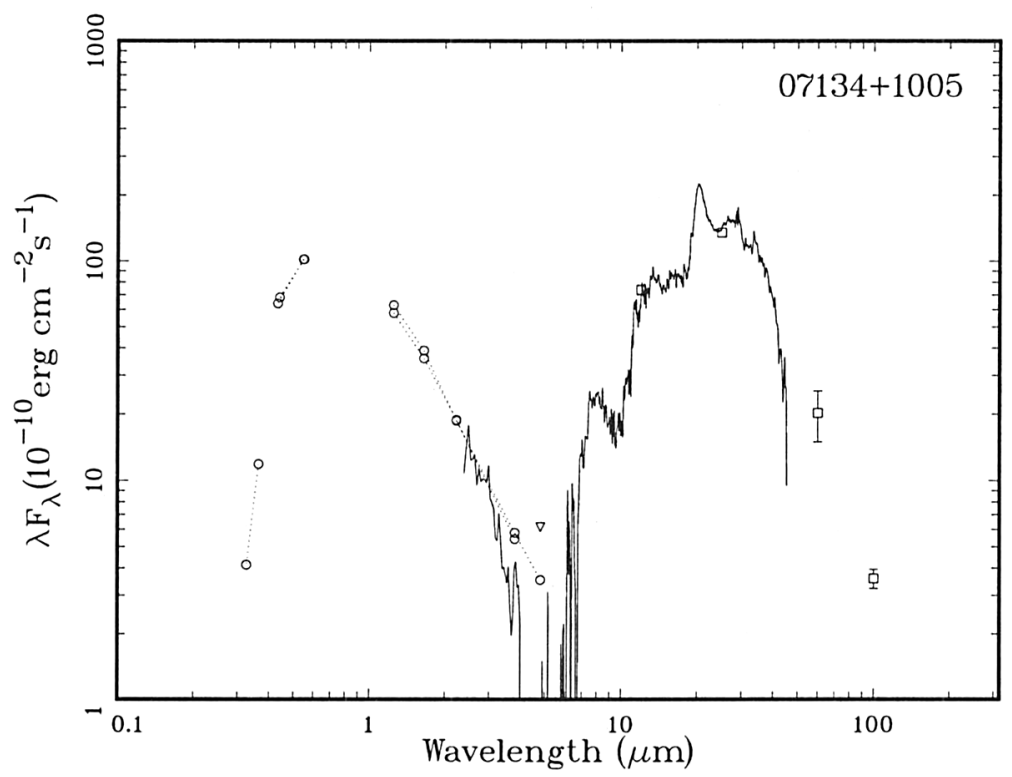

Figure 1. The spectral energy distribution of a typical $21 \mu \mathrm{m}$ source. The high temperature component is the reddened photosphere and the low temperature component is the remnant dust envelope from the AGB phase.

Table 1. List of $21 \mu \mathrm{m}$ sources and their common properties

\begin{tabular}{|c|c|c|c|c|c|c|}
\hline Name & $21 \mu \mathrm{m}$ & $30 \mu \mathrm{m}$ & Sp. Ty. & Optical & PAH features & Mol. lines \\
\hline $02229+6208$ & medium & & G8-K00-Ia & $\mathrm{C}_{2}, \mathrm{C}_{3}$ & & CO \\
\hline $04296+3429$ & strong & $\ldots$ & Go Ia & $\begin{array}{r}\mathrm{C}_{2}, \mathrm{C}_{3}, \\
\mathrm{CN}\end{array}$ & $\begin{array}{r}3.3,3.4-3.5 \\
7.7,11.3\end{array}$ & $\mathrm{CO}, \mathrm{HCN}$ \\
\hline $05113+1347$ & medium & & G8 Ia & $\mathrm{C}_{2}, \mathrm{C}_{3}, \mathrm{CN}$ & $3.3,11.3$ & $\mathrm{CO}$ \\
\hline $07134+1005$ & v. strong & medium & F5 I & $\mathrm{C}_{2}, \mathrm{CN}$ & $3.3,6.9$ & $\mathrm{CO}, \mathrm{HCN}$ \\
\hline $16594-4656$ & strong & 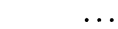 & & & .. & $\mathrm{CO}$ \\
\hline $19500-1709$ & v. weak & $\ldots$ & F3 I & . & $\ldots$ & $\mathrm{CO}, \mathrm{HCN}$ \\
\hline $20000+3239$ & weak & v. strong & G8 Ia & $\mathrm{C}_{2}, \mathrm{CN}$ & $7.7,11.3$ & $\mathrm{CO}, \mathrm{HCN}$ \\
\hline AFGL 2688 & weak & medium & F5 Iae & $\mathrm{C}_{2}, \mathrm{C}_{3}, \mathrm{CN}$ & $3.3,3.4-3.5$ & $\mathrm{CO}, \mathrm{HCN}$ \\
\hline $22223+4327$ & medium & & Go Ia & $\mathrm{C}_{2}, \mathrm{C}_{3}, \mathrm{CN}$ & & $\mathrm{CO}, \mathrm{HCN}$ \\
\hline $22272+5435$ & strong & v. strong & G5 Ia & $\mathrm{C}_{2}, \mathrm{C}_{3}$, & $\begin{array}{r}3.3,3.4-3.5 \\
6.9,7.7,11.3\end{array}$ & $\begin{array}{r}\mathrm{CO}, \mathrm{HCN}, \\
\mathrm{CS}\end{array}$ \\
\hline $22574+6609$ & medium & & & & $7.7,11.3$ & $\mathrm{CO}$ \\
\hline $23304+6147$ & v. strong & v. strong & G2 Ia & $\mathrm{C}_{2}, \mathrm{C}_{3}, \mathrm{CN}$ & $7.7,11.3$ & $\mathrm{CO}, \mathrm{HCN}$ \\
\hline
\end{tabular}




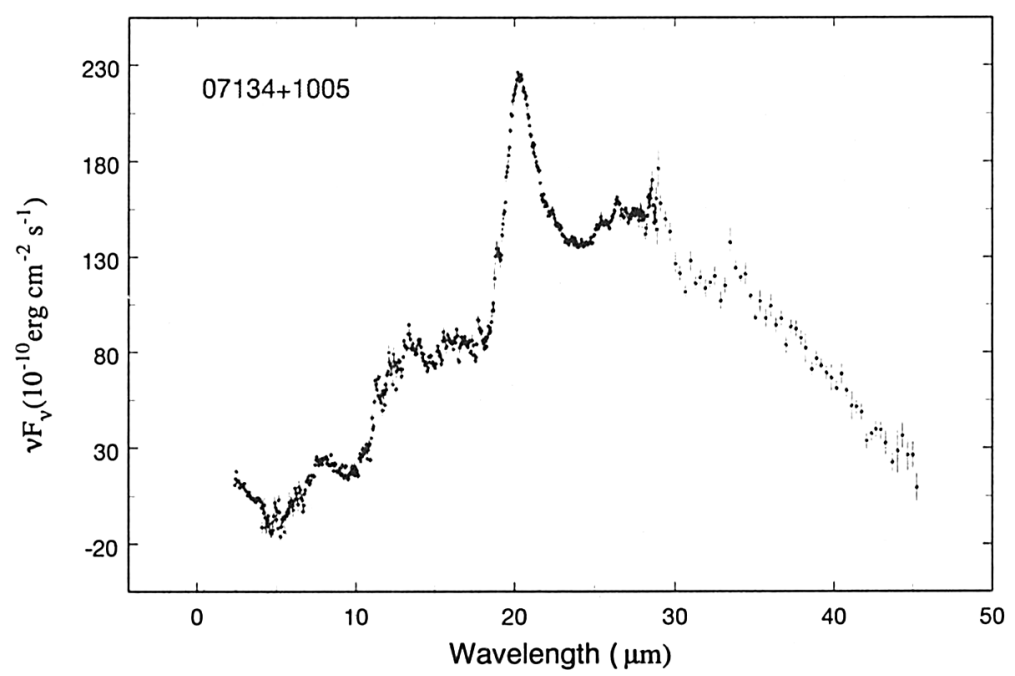

Figure 2. ISO SWS01 spectrum of IRAS $07134+1005$.

\section{The post-AGB nature of $21 \mu \mathrm{m}$ sources}

The post-AGB nature of the $21 \mu \mathrm{m}$ sources is evident from (1) their intermediate spectral types (F-G); (2) the red IRAS colors (intermediate between the reddest AGB stars and PN); (3) the presence of $\mathrm{C}_{2}$ and CN (Hrivnak 1995; Bakker et al. 1997) absorption bands consistent with large carbon enrichment through the 3rd dredge-up process; (4) excess of s-process elements (Van Winckel, this volume); and (5) their "double-peaked" spectral energy distributions, characteristic of dust shells detached from the stellar photospheres, implying the termination of mass loss sometime in the past (Fig. 1). These properties make the $21 \mu \mathrm{m}$ sources the most unambiguous PPN objects.

\section{ISO observations of $21 \mu \mathrm{m}$ sources}

Fig. 2 shows a typical infrared spectrum of a $21 \mu \mathrm{m}$ source. Other than the strong, broad emission feature at $21 \mu \mathrm{m}$, the spectrum shows an almost flat continuum between 12 and $18 \mu \mathrm{m}$, and another broad feature between 11 and $13 \mu \mathrm{m}$. The sharp feature at $11.3 \mu \mathrm{m}$ is likely to be associated with the C-H outof-plane bend of PAH molecules. An unidentified, very broad emission feature at $30 \mu \mathrm{m}$ is also often found in $21 \mu \mathrm{m}$ sources.

The $21 \mu \mathrm{m}$ features have almost exactly the same shape in each source after continuum subtraction. Fig. 3 shows the profiles of the $21 \mu \mathrm{m}$ feature of 3 different sources at the higher resolution SWS06 mode of ISO $(\Delta \lambda / \lambda \sim 500)$. The feature spans 19 to $23 \mu \mathrm{m}$ with no obvious narrow substructures. 


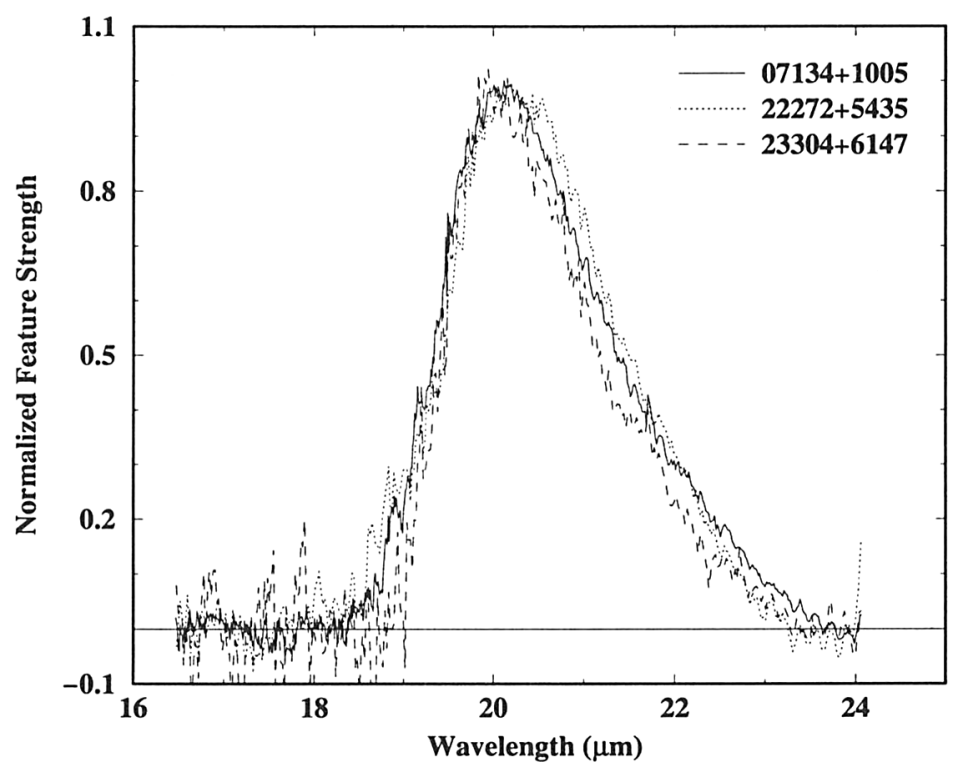

Figure 3. Overlay of the ISO SWS06 spectra of three $21 \mu \mathrm{m}$ features.

\section{Relationship with the $30 \mu \mathrm{m}$ feature}

The detection of a $30 \mu \mathrm{m}$ emission feature in four $21 \mu \mathrm{m}$ sources $(07134+1005$, $20000+3239,22272+5435$ and $23304+6147$; Omont et al. 1995) suggests that these two features may share a common origin. However, the $30 \mu \mathrm{m}$ feature has been seen in AGB stars (e.g. IRC +10216) and PN (e.g. IC 418) as well as in PPN (Cox 1993), while the $21 \mu \mathrm{m}$ feature thus far appears to be restricted to PPN.

\section{Relationship with the PAH molecule}

There is strong evidence that the $21 \mu \mathrm{m}$ feature is related to the PAH molecules. The 3.4-3.5 $\mu \mathrm{m}$ emission is particularly strong in $21 \mu \mathrm{m}$ sources (Geballe et al. 1992). However, the 3.4-3.5 $\mu \mathrm{m}$ feature is absent in the strongest $21 \mu \mathrm{m}$ emission source (IRAS $07134+1005$; Kwok et al. 1990), so the correlation is not perfect.

The 3.4-3.5 $\mu \mathrm{m}$ feature has been suggested to be due to the hot bands of the fundamental $3.3 \mu \mathrm{m}$ (CH stretch) feature (Barker et al. 1987), although this cannot be the case in PPN because the 3.4-3.5 $\mu \mathrm{m}$ feature is almost as strong as the $3.3 \mu \mathrm{m}$ feature. More possibly, the $3.4-3.5 \mu \mathrm{m}$ feature is due to aliphatic sidegroups $\left(-\mathrm{CH}_{2},-\mathrm{CH}_{3}\right)$ attached to the PAH molecules (Jourdain de Muizon et al. 1989).

The $6.9 \mu \mathrm{m}$ feature is not seen in PN but is strong in $21 \mu \mathrm{m}$ sources. It is therefore possible that the 3.3-3.4, 6.9, and $21 \mu \mathrm{m}$ sources are related. However, IRAS $07134+1005$ has a strong $6.9 \mu \mathrm{m}$ feature but not the $3.4 \mu \mathrm{m}$ feature. 


\section{Origin of the $21 \mu \mathrm{m}$ feature}

Many candidates have been proposed as possible carriers of the $21 \mu \mathrm{m}$ feature, including:

- amides: urea or thiourea (Sourisseau et al. 1992)

- hydrogenated amorphous carbon (HAC; Buss et al. 1990)

- hydrogenated fullerenes $\left(\mathrm{C}_{60} \mathrm{H}_{m}, \mathrm{~m}=1,2, \ldots 60\right.$, or fulleranes; Webster 1995)

- maghemite $\left(\mathrm{Fe}_{2} \mathrm{O}_{3}\right)$ or magnetite $\left(\mathrm{Fe}_{3} \mathrm{O}_{4} ; \mathrm{Cox} 1990\right)$

- solid $\mathrm{SiS}_{2}$ (Nuth et al. 1985; Goebel 1993; Begemann et al. 1996)

In view of the fact that the $21 \mu \mathrm{m}$ feature is seen exclusively in carbon-rich objects, it seems unlikely that it is due to inorganic materials such as $\mathrm{SiS}_{2}$ or $\mathrm{Fe}_{2} \mathrm{O}_{3}$. The correlation of the $21 \mu \mathrm{m}$ feature with the PAH features suggests that its carrier is a large carbon-based molecule. Most PAH molecules have ring deformation vibration modes around $20 \mu \mathrm{m}$, and mid-infrared laboratory emission spectroscopy of simple PAH molecules such as naphthalene, pyrene, and chrysene shows broad emission features in that region ( $P$. Bernath, private communication).

Considering that fullerenes $\left(\mathrm{C}_{60}\right)$ are the $3-\mathrm{D}$ analogues of the 2-D PAH molecules, their existence in carbon-rich objects is not unexpected. Emission spectra of gas-phase $\mathrm{C}_{60}$ show strong features at $7.1,8.6,17.5$, and $19 \mu \mathrm{m}$ (Frum et al. 1991), whereas the lowest vibrational mode of $\mathrm{C}_{60} \mathrm{H}_{60}$ is calculated to be at $23 \mu \mathrm{m}$ (Webster 1995). For the other unsaturated hydrides $\left(\mathrm{C}_{60} \mathrm{H}_{m}\right.$, $\mathrm{m}=1$ to 59 ), the perfect symmetry of the molecule is broken and the mode is no longer a narrow feature but bands peaking at wavelengths from 19 to $23 \mu \mathrm{m}$ as $m$ changes from 0 to 60 . Webster (1995) suggests that the observed broad $21 \mu \mathrm{m}$ feature is the result of a mixed population of fulleranes.

\section{Evolutionary scenario}

As a star evolves from the AGB to PN, its circumstellar environment (density, temperature, and radiation background) undergoes drastic changes. The dominant infrared emission features will likely change as the conditions for molecular formation, destruction, and excitation change. Acetylene $\left(\mathrm{C}_{2} \mathrm{H}_{2}\right)$, widely considered to be the first building block of $\mathrm{PAH}$ molecules, has recently been detected in the atmospheres of carbon stars (Yamamura et al., this volume). Thus it is possible that PAH molecules are synthesized in AGB stars, but that the molecules are not excited because of the low temperatures of the central stars. HAC grains, made up of aromatic rings of various sizes bonded peripherally to polymeric or hydrocarbon species (Duley \& Willams 1986), can also be made during the late AGB phase. Extreme carbon stars on the AGB have featureless dust continua (Volk et al. 1992) and these could be due to HAC grains. During the transition from the AGB to PN, these grains may be gradually shattered into smaller PAH molecules, possibly by high-velocity outflows now observed in 
PPN. Then as the UV radiation background increases as the central star evolves to higher temperatures, the strengths of the PAH features increase. If $21 \mu \mathrm{m}$ features are due to $\mathrm{HAC}$ grains, then the destruction of $\mathrm{HAC}$ grains will also imply the disappearance of the $21 \mu \mathrm{m}$ feature beyond the PPN phase.

If the $21 \mu \mathrm{m}$ feature is related to fulleranes and the disappearence of the $21 \mu \mathrm{m}$ feature is the result of the loss of $\mathrm{H}$ atoms, then fullerenes should be detected in PN if they can be excited by UV photons or collisions. The infrared features of $\mathrm{C}_{60}$ have been searched in $\mathrm{R} \mathrm{CrB}$ and IRC +10216 without success (Clayton et al. 1995), but a more extensive search in $\mathrm{PN}$ may be fruitful.

\section{References}

Bakker E.J., van Dishoeck E.F., Waters L.B.F.M., Schoenmaker T., 1997, A\&A 323,469

Barker J.R., Allamandola L.J., Tielens A.G.G.M., 1987, ApJ 315, L61

Begemann B., Dorschner J., Henning Th., Mutschke H., 1996, ApJ 464, L195

Buss R.H., Cohen M., Tielens A.G.G.M., Werner M.W., Bregman J.D., Rank

D., Witteborn F.C., Sandford S., 1990, ApJ 365, L23

Clayton G.C., Kelly D.M., Lacy J.H., Little-Marenin I.R., Feldman P.A., Bernath P.F., 1995, AJ 109, 2096

Cox P., 1990, A\&A 236, L29

Cox P., 1993, in Astronomical Infrared Spectroscopy, S. Kwok (ed.), ASP Conf. Ser. 41, p. 163

Duley W., Willams D.A., 1986, MNRAS 219, 859

Frum C.I., Engleman R., Hedderich H.G., Bernath P.F., Lamb L.D., Huffman D.R., 1991, Chem. Phys. Let. 176, 504

Geballe T.R., Tielens A.G.G.M., Kwok S., Hrivnak B.J., 1992, ApJ 387, L89

Goebel J.H., 1993, A\&A 278, 226

Hrivnak B.J., 1995, ApJ 438, 341

Jourdain de Muizon M., Cox P., Lequeux J., 1989, A\&AS 83, 337

Justtanont K., Barlow M.J., Skinner C.J., Roche P.F., Aitken D.K., Smith C.H., 1996, A\&A 309, 612

Kwok S., Hrivnak B.J., Geballe T.R., 1990, ApJ 360, L23

Kwok S., Hrivnak B.J., Geballe T.R., 1995, ApJ 454, 394

Kwok S., Volk K., Bidelman W.P., 1997, ApJS 112, 557

Kwok S., Volk K., Hrivnak B.J., 1989, ApJ 345, L51

Nuth J.A., Moseley S.H., Silverberg R.F., Goebel J.H., Moore W.J., 1985, ApJ 290, L41

Omont A., et al., 1995 ApJ 454, 819

Sourisseau C., Coddens G., Papoular R., 1992, A\&A 254, L1

Volk K., Kwok S., Langill P.P., 1992, ApJ 391, 285

Webster A., 1995, MNRAS 277, 1555 